\title{
A Segmentation-Based Regularization Term for Image Deconvolution
}

\author{
Max Mignotte
}

\begin{abstract}
This paper proposes a new and original inhomogeneous restoration (deconvolution) model under the Bayesian framework for observed images degraded by space-invariant blur and additive Gaussian noise. In this model, regularization is achieved during the iterative restoration process with a segmentation-based a priori term. This adaptive edge-preserving regularization term applies a local smoothness constraint to pre-estimated constant-valued regions of the target image. These constant-valued regions (the segmentation map) of the target image are obtained from a preliminary Wiener deconvolution estimate. In order to estimate reliable segmentation maps, we have also adopted a Bayesian Markovian framework in which the regularized segmentations are estimated in the maximum a posteriori (MAP) sense with the joint use of local Potts prior and appropriate Gaussian conditional luminance distributions. In order to make these segmentations unsupervised, these likelihood distributions are estimated in the maximum likelihood sense. To compute the MAP estimate associated to the restoration, we use a simple steepest descent procedure resulting in an efficient iterative process converging to a globally optimal restoration. The experiments reported in this paper demonstrate that the discussed method performs competitively and sometimes better than the best existing state-of-the-art methods in benchmark tests.
\end{abstract}

Index Terms-Adaptive prior model, Bayesian estimation, image deconvolution or restoration, image segmentation, Markovian model, Tikhonov regularization.

\section{INTRODUCTION}

\section{A. Problem Statement}

$\mathbf{T}$ HE problem of recovering an original image $x$ from a degraded observed version $y$, for the purpose of improving its quality or obtaining some type of information that is not readily available from the degraded image [1] is usually known as a restoration or reconstruction problem. In many situations where the imaging system is assumed to be linear and shift invariant, the transformation from $x$ to $y$ is well described by the following and familiar additive linear degradation model

$$
y=h * x+n
$$

where $y, x$, and $n$ represent, respectively, the degraded or noisy and blurred observed image (of size $N$ pixels), the undistorted true image and the corrupting additive and white Gaussian noise with variance $\sigma^{2} . h$ is the point spread function (PSF) of the

Manuscript received January 26, 2005; revised August 15, 2005. The associate editor coordinating the review of this manuscript and approving it for publication was Dr. Mario A. T. (G. E.) Figueiredo.

The author is with the Département d'Informatique et de Recherche Opérationnelle (DIRO), Université de Montréal, Montréal, QC H3C 3J7 Canada (e-mail: mignotte@iro.umontreal.ca).

Digital Object Identifier 10.1109/TIP.2006.873446 imaging system and $*$ is the linear convolution operator. We shall assume throughout this paper that the degradation model (PSF and variance of the white Gaussian noise) is known. It might be given analytically or given numerically based on previous estimations or calibration experiments.

The simplest way to approach the restoration problem is to find $x$ in the maximum likelihood (ML) sense (according to the statistics of the stationary Gaussian noise $n$ ) or equivalently for this problem, to find the least squares estimation of $x$ defined by

$$
\begin{aligned}
\hat{x}_{\mathrm{ML}} & =\arg \max _{x} P_{Y \mid X}(y \mid x) \\
& =\arg \max _{x} \exp \left\{-\frac{1}{2} \beta\|y-h * x\|^{2}\right\} \\
& =\arg \min _{x}\|y-h * x\|^{2} .
\end{aligned}
$$

This so-called inverse-filtering solution which best matches the probabilistic behavior of the data leads to unacceptable restoration solutions. This is due to the fact that several candidate images can be a solution of (2). In addition to the ideal image, others are noisy versions of $x$ and the others do not have many of the properties expected in the original image $x$. In fact, the problem of recovering an original image from its noisy and degraded version is typical of ill-posed inverse problems in the sense of Hadamard [2]: The perfect knowledge of the degradation model is not sufficient to determine a restoration result with acceptable accuracy. To circumvent this difficulty, a conventional method is to regularize the solution by introducing a priori constraints [3]. This regularization constraint can be expressed as the prior distribution of the unknown image $x$ (treated as a realization of a random field $X$ ), within a Markov random field (MRF) framework [3], [4]. The prior distribution reflects the knowledge or beliefs concerning the types of images acceptable as estimates and regularizes the optimization problem so that a unique solution always exists [5] (i.e., a well-posed problem).

The knowledge concerning the types of images acceptable as estimates can be encoded as follows.

- Analytically. In this way, the encoding is done in the regularization framework, through an energy function $\Omega(x)$ added to the likelihood term $\|y-h * x\|^{2}$.

- From the probabilistic viewpoint, via a prior distribution of the form

$$
P_{X}(x) \propto \exp \left\{-\frac{1}{2} \gamma \Omega(x)\right\} .
$$

In this framework, a regularized solution corresponds to the maximum A posteriori (MAP) solution, i.e., the 
solution $\hat{x}_{\mathrm{MAP}}$ that maximizes the posterior distribution

$$
\begin{aligned}
P_{X \mid Y}(x \mid y) & \propto P_{X}(x) P_{Y \mid X}(y \mid x) \\
\hat{x}_{\mathrm{MAP}} & =\arg \max _{x} P_{X \mid Y}(x \mid y) \\
& =\arg \min _{x}\left\{\|y-h * x\|^{2}+\frac{\alpha}{2} \Omega(x)\right\} .
\end{aligned}
$$

The criterion expressed by (4) (also called the penalized maximum likelihood) contains two terms, the first expresses the fidelity to the available data $y$ and the second encodes the property expected in the true undegraded image. $\alpha / 2=\gamma / \beta$ is the regularization parameter controlling the contribution of the two terms.

\section{B. Existing Prior Models}

Probably the simplest prior model used to regularize the solution is $\Omega(x)=\|x\|^{2}$ which yields to the so-called Tikhonov regularization with the identity [6], or equivalently to the inverse Wiener filter (in this case, $\alpha / 2$ is a constant approximating the ratio between the noise and the Fourier spectrum of $x$, see Section III-A). By using this prior, we penalize solutions exhibiting large variance in its gray level distribution. This regularization strategy often induces spurious oscillations or ringing occurring at discontinuities (or edges) of the image and thus undesirable restoration results.

A commonly used choice is the quadratic functional $\Omega(x)=\|C * x\|^{2}$ where $C$ is a PSF associated to a high-pass filter (gradient or Laplacian). This penalty function penalizes solutions with large high-frequency fluctuations and thus enforces smoothness on the solution. A similar strategy reinterpreted in the wavelet-domain has been proposed by Wang et al. in [7] with the following quadratic functional, $\Omega(x)=\sum_{j \geq J_{1}} \sum_{k} b_{j}\left\|\theta_{j, k}\right\|^{2}$, where $\theta_{j, k}$ are wavelet coefficients of $x$ at resolution level $j$ and $b_{j+1} \geq b_{j}>0$ are constants (i.e., the larger the resolution level, the larger the penalty). A similar prior model, but adaptive to the subband decomposition of $x$, can be found in [8].

In the same way, another prior model, which can be used to model global smoothness of the desired restoration, is the conditional autoregression (CAR) model defined by Ripley [9] in which $\Omega(x)=\sum_{s \in S}\left(x_{s}-\phi \sum_{\langle s, t\rangle} x_{t}\right)^{2}$ where the notation $\langle s, t\rangle$ denotes the four neighbor pixels at distance one from pixel $x_{s}$ at location $s$.

As an alternative to the quadratic regularization terms described above, some authors have also proposed the use of complexity penalty terms. It is an interesting way to penalize roughness which borrows from recent developments in nonparametric estimation theory. The theoretical motivation for complexity regularization is explained in detail in [10]. In this regularization approach, estimates with high complexity in a data-compression sense are penalized [11]. This complexity is generally measured by the length of a binary string used to encode the restored image [12]. Complexity regularization includes maximum description length (MDL) as a special case [13]. As there exists a great variety of compression schemes, the complexity regularization term may take diverse forms (see [11] for penalties associated with state-of-the-art coders). For instance, we can cite the simple complexity penalty
$\Omega(x)=(3 / 2) M \log _{2} N$, where $M$ is the number of nonzero wavelet coefficients. Another way consists in exploiting the sparsity of the wavelet coefficients of the signal to be estimated. This property expected in the original image is well modeled by a function of the form $\Omega(x)=|b \theta|^{\nu}$ (leading to generalized Gaussian prior density) where $\theta$ is the wavelet coefficient decomposition of $x$ and $\nu$ is the so-called shape parameter (typically between 0.5 and 1 ). A variant is proposed in [14] where the parameter $b$ is different for the set of wavelet coefficients at different scales and another is proposed in which the distribution of wavelet coefficients is based on mutually independent Gaussian mixture models [15].

Among the other wavelet-based restoration methods, we can cite the hidden Markov tree (HMT) prior model using a multiscale Gaussian mixture of complex wavelet coefficients proposed in [16], [17], a decreasing exponential law [18] (i.e., a low complexity penalty term), or a Gaussian scale mixture [19]. To compute the MAP estimate, they developed an expectation maximization (EM) [20] where the missing variables are respectively, the variance of each Gaussian of the considered mixture [17], the deblurred but noisy image [18] and the scale factors of the prior Gaussian mixtures [19].

Another regularizing term is the total variation functional denoted by $\Omega(x)=|\nabla x|_{1}$, where $\nabla x$ denotes the gradient of $x$. In this context, the prior distribution $P_{X}(x) \propto \exp (-\Omega(x))$ describes images to be recovered as consisting largely of zero gradient regions interspersed with occasional strong gradient transitions. Theoretical analysis [21], [22] has demonstrated the superiority of total variation (TV) criteria over quadratic regularization when the solution is blocky (i.e., piecewise smooth) and its efficiency for recovering edges of images. This approach can be viewed as a special case of the "half-quadratic regularization" scheme of Geman et al. [23], [24] and of the ARTUR model proposed by Charbonnier et al. [25].

These nonquadratic penalties and non Gaussian priors can provide better restorations with good edge-preserving properties, but this class of methods is often more computationally expensive. Let us also add that some of the multiscale waveletbased restoration methods are analogous to classical Wiener filters (e.g., [26]), TV variational methods, or some Tikhonov regularization procedures reinterpreted in the wavelet-domain. These methods may have computational advantages, but do not overcome the major drawbacks associated with a linear filtering method (e.g., over-smoothing). Besides, these models are homogeneous and assume that the original image to be recovered is smooth. However, this is a global requirement and therefore not very effective in terms of local smoothness. A more efficient image model assumes that only homogeneous regions are smooth, and that edges must remain sharp. In other words, a good prior model should adapt to the local characteristics and structure of the image to enable the solution to be less noisy in constant areas and to exhibit sharper details in other regions.

\section{Spatially Adaptive Techniques Based on Quadratic Penalties or a Line-Process}

In this way, spatially adaptive techniques to the restoration problem, based on quadratic penalties or a line-process model, have been proposed. Among them, we can cite the seminal work 
of Geman and Geman with their MRF model with line processes [3] and the doubly stochastic MRFs of Woods et al. [27]. However, using line elements adds to the complexity of the problem by increasing both the dimensionality of the required optimization and the complexity of the parameter estimation procedure (required to make the restoration unsupervised). Another technique, also proposed by Geman and Reynolds, to model local smoothness constraints without introducing auxiliary variables is given in [23]. This technique allows the recovery of discontinuities with a regularization function of the form $\Omega(x)=$ $\sum_{C} \phi\left(D_{C}^{k}(x) / \Delta\right)$, where $\phi(u)=-\left(1+|u|^{\gamma}\right)^{-1}$ and the first summation is over all horizontal and vertical nearest neighbor pairs of pixels, and $D_{C}^{k}(x)$ is simply the difference $x_{s}-x_{t}$. In the context of edge-preserving restoration techniques, we can also cite the significant contribution of Bouman and Sauer in [28] with their generalized Gaussian MRF prior model of the form $\Omega(x) \propto-\gamma^{q} \sum_{\langle s, t\rangle} \beta_{s t}\left|x_{s}-x_{t}\right|^{q}$ where $1 \leq q \leq 2$ is a parameter that controls the smoothness of the image model and/or the sharpness of the edges to form in the restored image. This model includes a Gaussian MRF for $q=2$, and an absolute-value potential function with $q=1$ also referred to as the "median pixel prior." We can also cite the adaptive Gaussian model proposed in [29] which allows to locally adjust the amount of regularization, with the following quadratic energy: $\Omega(x)=$ $\sum_{s \in S} b_{s}^{x}\left(D_{x} x_{s}\right)^{2}+b_{s}^{y}\left(D_{y} x_{S}\right)^{2}$ where $b_{s}^{x}$ and $b_{s}^{y}$ are the adaptive parameters and $D_{x}, D_{y}$ are first order derivative operators. The variables $b$ are analogous to a continuous line process [3]: a low value of $b$ corresponding to an edge between two pixels. Let us also add the interesting and simple approach developed by Banham and Katsaggelos [30] which simply consists in switching the linear inverse filter spatially based on an edge detection test, enabling the overall procedure to automatically preserve details near detected edges.

\section{Proposed Approach}

An alternative approach to apply smoothness constraint, while preserving discontinuities, is to apply a smoothness constraint only on constant areas of the image to be recovered. This problem of identifying constant regions in a given image is a low-level task in image processing and is called a segmentation problem. By assuming that a good oversegmentation of the image to be recovered is estimated, a partition into homogeneous regions can be obtained by simply searching the set of disjoint regions (i.e., the set of connected pixels belonging to the same class). A simple and adaptive local quadratic smoothness term would then consist in penalizing solutions exhibiting a luminance distribution with large variance within each regions. This regularization strategy, which assumes as prior model that the image is piecewise smooth over pre-estimated regions is the one proposed in this paper.

Therefore, in contrast with almost all recent edge-preserving contributions in image restoration, manipulating "edge variables" in the regularization term, we herein propose instead to use "label variables" taken into account by a preliminary segmentation process.

In order to extract a reliable oversegmentation map (robust to the noise), we have adopted a statistical framework allowing to estimate the solution in the MAP sense. Nevertheless, the problem of this simple approach is obviously twofold:

- The first difficulty is that the restored image is required for the segmentation while the segmentation is also required to constrain the restoration procedure. To circumvent this difficulty, a simple scheme would consist in implementing alternatively the restoration and the segmentation procedure until convergence. In our application, this segmentation is simply obtained on a rough restoration. To this end, we use the result of an iterative Wiener filter [31], both to estimate the oversegmentation map, but also to initialize our iterative procedure of restoration. Eventually, the final result of restoration can be used to re-estimate a better segmentation and to refine, in a second pass, the restoration result.

- The segmentation problem can also be seen as the solution of an inverse ill-posed problem as expressed in [3]; i.e., the data in themselves are insufficient to unambiguously define the segmentation. In order to rightly constrain the nature of this problem, some particular knowledge about the scene is necessary. A simple a priori knowledge may express the fact that nearby pixels are fairly likely to belong to the same class. In a probabilistic framework, such regularities are well captured by a MRF prior model [4]. Thanks to the Hammersley-Clifford theorem, this prior model can be parametrically described by a Gibbs distribution based on spatially local interactions as the standard Potts prior model.

Another difficulty is that the generic problem of unsupervised Markovian segmentation is quite complex and remains an active domain of research in the low-level vision community. The main difficulty is that the estimation of parameters is required for the segmentation, while one (or several) segmentation(s) are usually required for parameter estimation. To circumvent this difficulty, we have adopted the monoscale version of the Markovian segmentation model described in [32] already successfully applied to noisy sonar images. In this approach, the unsupervised MRF-based segmentation problem consists in having a two-step process. First, a parameter estimation step is conducted to infer the noise model parameters, (thanks to an iterative method called iterative conditional estimation or ICE). Then, a second step is devoted to the segmentation itself based on the values of estimated parameters. Let us add that the segmentation is computed on the result of an inverse filtering, i.e., on an noisy image widely deblurred. In this situation, the Markovian segmentation procedure remains well suited to take into account this noise degradation in its likelihood model.

In this way, the proposed restoration is performed by using a two-level MRF model. The first one includes a low-level prior model used in the segmentation procedure and, more precisely, a simple Potts prior model ensuring homogeneity of the segmentation map and a likelihood model expressing the luminance distribution of each homogeneous region of the image. While the second exploits the segmentation result, and more precisely a partition into regions extracted from this segmentation map as a meta parameter of our prior model of restoration. This prior model efficiently expresses the expected local smoothness property in the image to be recovered. The restoration procedure 
finds the best solution that is consistent to the data, i.e, which blurred values are close to $y$ (likelihood model) and that respects this local smoothness property. These two estimation problems (segmentation and restoration) are defined as the search of the MAP or equivalently as an optimization problem in which the posterior energy has to be minimized. To this end, we use a gradient-based algorithm, more precisely an ICM algorithm for the segmentation step and a steepest descent procedure for the restoration step.

\section{E. Paper Organization}

This paper is organized as follows. The proposed spatially adaptive restoration model is described in Section II. In Section III, we detail the parameter estimation step of our prior model, i.e., the iterative Wiener Filter used to obtain a rough restoration and the unsupervised Markovian segmentation model. Finally, Section IV presents a set of experimental results and comparisons with existing techniques.

\section{Proposed AdAPtive EdGE-PRESERVING RESTORATION MODEL}

In order to impose local smoothness on constant areas of the image to be restored, we propose the following quadratic regularization term, based on one or several simulated partitions into regions and whose goal is to penalize solutions exhibiting a luminance distribution with large variance within each region

$$
\Omega(x)=\|\rho(x)\|^{2}=\left\|x-\frac{1}{N_{\text {Seg }}} \sum_{k=1}^{N_{\text {Seg }}} \Gamma_{k}(x)\right\|^{2}
$$

where $N_{\text {Seg }}$ is the number of partitions into regions. $\Gamma_{k}\left(x_{s}\right)$ designates the operator that gives the mean of gray level values of the region of belonging to the pixel at location $s$ (for the $k^{\text {th }}$ simulated partition into regions).

The interest of doing several partitions into regions, in fact one partition for each simulation of a segmentation map, is to minimize the effects of the dependence of a bad segmentation map on the restoration result. More precisely, the interest is to minimize the effects of possible bad classification and localization that could occur in the case of only one segmentation on certain low-contrasted, very noisy or highly textured portions of the image. This strategy will be also discussed and quantified (in Section IV) in the ISNR sense for several degradation models.

In this context, the restoration problem is thus defined as the search of the global minima of the following energy function

$$
E(x)=\left\{\|y-h * x\|^{2}+\frac{\alpha}{2}\left\|x-\frac{1}{N_{\text {Seg }}} \sum_{k=1}^{N_{\text {Seg }}} \Gamma_{k}(x)\right\|^{2}\right\} .
$$

This search is performed, in our application, by a steepest descent procedure which moves the estimates iteratively in the negative gradient direction, as follows:

$$
\hat{x}^{[n+1]}=\hat{x}^{[n]}-\gamma \nabla E(x)
$$

where $\gamma$ is the step size. A large step size $\gamma$ is needed for fast convergence, but a too large value may destabilize the iterative algorithm. $\nabla E(x)$ with $E(x)$ defined by (6) can be easily defined and allows to obtain the following iterative procedure of restoration

$$
\hat{x}^{[n+1]}=\hat{x}^{[n]}+\gamma\left(h^{\#} *\left(y-h * x^{[n]}\right)-\alpha \rho^{\prime}\left(\hat{x}^{[n]}\right) \rho\left(\hat{x}^{[n]}\right)\right)
$$

where $h^{\#}(i, j)=h(-i,-j)$ (the coordinates $(i, j)$ represent the discrete pixel locations and for $h$ symmetric, we have $h^{\#}=$ $h), *$ is the linear convolution operator, and, in this form of notation, the multiplication (between $\rho^{\prime}$ and $\rho$ ) is done point-bypoint (or pixel-by-pixel). Besides, the image is assumed to be toroidal.

Let us now find an analytical expression for $\rho^{\prime}$. To this end, let us assume that $N_{\text {Seg }}=1$ (one partition into regions). If $\mu$ denotes the mean of the gray level values of the region to which the pixel at location $s$ belongs, we have

$$
\begin{aligned}
\frac{\partial \rho(x)}{\partial x_{s}} & =\lim _{\epsilon \rightarrow 0} \frac{\rho\left(x_{s}+\epsilon\right)-\rho\left(x_{s}\right)}{\epsilon} \\
& =\lim _{\epsilon \rightarrow 0}\left\{\frac{\left(x_{s}+\epsilon-\frac{\mathcal{N} \mu+\epsilon}{\mathcal{N}}\right)-\left(x_{s}-\mu\right)}{\epsilon}\right\} \\
& =1-\frac{1}{\mathcal{N}}
\end{aligned}
$$

where $\mathcal{N}$ is the number of pixels of the region belonging to pixel at location $s$. In the case where several partitions into regions are considered, we can easily find

$$
\frac{\partial \rho(x)}{\partial x_{s}}=1-\frac{1}{N_{\text {Seg }}} \sum_{k=1}^{N_{\text {Seg }}} \frac{1}{\mathcal{N}_{k}}
$$

where $\mathcal{N}_{k}$ is the number of pixels of the region belonging to pixel at location $s$ for the $k^{\text {th }}$ simulated partition into regions.

The successive approximation of the solution according to the minimization of the cost function expressed in (6) results in initializing $\hat{x}^{[0]}$ with the restoration result given by the Iterative Wiener filter (see Section III-A), and by using the iterative process defined in (8) [with the use of (9)] until some convergence criterion is met.

A conjugate gradient technique could be efficiently applied to speed up the convergence of the proposed gradient-based iterative restoration algorithm. An interesting and alternative approach will also be to consider a coordinate-wise (local) descending algorithm, e.g., a Jacobi or Gauss-Seidel iterative procedure which would avoid the tuning of the step size parameter $\gamma$ (as an overrelaxed version of this steepest gradient procedure could also do it). Besides, since the proposed criterion is quadratic, many other optimization methods can be used.

Let us finally add that, in this context, the proposed iterative restoration approach is not purely Bayesian in the sense that the segmentation-based prior is estimated from data.

\section{PARAmeter Estimation Step of Prior Model}

The proposed restoration algorithm assumes knowledge of an oversegmentation of the original image $x$ into homogeneous regions. Since this image (before degradation) is unknown, the first step of our algorithm consists of obtaining an approximation of the true image $x$, with an unsupervised Wiener filter, 
using the iterative technique described in [31]. This procedure is briefly recalled here.

\section{A. Iterative Wiener Filter}

Knowing the degradation model (blurring and noise functions), Wiener filtering is often suggested as noniterative procedure allowing to obtain a first good approximation of the restoration result.

Nevertheless, in order to obtain a good restoration result optimal in the minimum mean-square error (MMSE) sense, an accurate knowledge of $|X(u, \nu)|^{2}$, the Fourier spectrum of $x$ (the ideal solution to be estimated) is required by this procedure. Usually, the Fourier spectrum of $x$ is approximated by the spectrum of the degraded image (which is sometimes far from the true spectrum of the ideal image due to the degradation model) and, consequently, the restoration filter is no longer MMSE optimal, leading to suboptimal (and undesirable) restoration results.

To circumvent this difficulty, an iterative procedure is suggested in [31] whereby successively restored images are used to update $|X(u, \nu)|^{2}$ and subsequently to improve the restoration result, namely

$$
X^{[n+1]}(u, \nu)=Y(u, \nu)\left[\frac{H^{\star}(u, \nu)}{|H(u, \nu)|^{2}+\frac{\sigma^{2}}{\left|X^{[n]}(u, \nu)\right|^{2}}}\right]
$$

with $X^{[0]}(u, \nu)=Y(u, \nu)$, and where $Y(u, \nu)$ and $H(u, \nu)$ represent the Fourier transform of the degraded image and the PSF respectively. Superscripts denote the iteration number of the procedure and $(.)^{\star}$ means the complex conjugate of $($.$) .$

Analysis in [31] shows that the procedure converges to a fixed point which is not the desired minimum MSE solution (except when $\sigma^{2}=0$ ). A correction term is also proposed in [31] to modify the iterative filter for the desired theoretical minimum MSE result where exact Fourier spectrum of $x$ is assumed

$$
\left|X^{[n+1]^{+}}(u, \nu)\right|^{2}=\left|X^{[n+1]}(u, \nu)\right|^{2}+\operatorname{Cor}^{[n+1]}
$$

with

$$
\operatorname{Cor}^{[n+1]}=\left|X^{[n]}(u, \nu)\right|^{2}-\frac{\left|X^{[n+1]}(u, \nu)\right|^{2}|H(u, \nu)|^{2}}{|H(u, \nu)|^{2}+\frac{\sigma^{2}}{\left|X^{[n+1]}(u, \nu)\right|^{2}}} .
$$

The correction is applied after each step of the iterative procedure defined in (10). The result $\left|X^{[n+1]^{+}}\right|^{2}$ is used in place of $\left|X^{[n+1]}\right|^{2}$ to estimate $X^{[n+2]}$ using (10), and the procedure is repeated until convergence is achieved (i.e., until $X^{[n+1]} \approx X^{[n]}$ and typically after 5-40 iterations).

However, as indicated in [19], since the estimation of $X(u, \nu)$, after convergence, is not reliable at frequencies where the blurring operator $H(u, \nu) \approx 0$ (e.g., in the case of a motion blur), we finally terminate the procedure by a last Wiener filtering in which $|X(u, \nu)|^{2}+\epsilon$ is used in place of $|X(u, \nu)|^{2}$ in order to boost the estimated $|X(u, \nu)|^{2}$ at high frequencies. In our application $\epsilon$ is set to $10^{-3}$.

Depending on the degradation model, the above-outlined corrected iterative Wiener filter yields restorations in which discontinuities are smoothed out and which suffer from spurious oscillations or ripples (see Section IV). This so-called Gibbs phenomenon is, in fact, due to the underlying Fourier basis elements whose support is over the entire spatial domain. Fig. 1(c) displays an example of restoration obtained with this procedure on a noisy and blurred CAMERAMAN image (uniform blur of size $9 \times 9$, and white Gaussian noise variance of $\sigma^{2}=0.308$ ensuring a BSNR $=40 \mathrm{~dB}$ ). Nevertheless, the quality of the restored image by this procedure is sufficient to automatically estimate a Markovian segmentation that will be used in our restoration model as meta parameter of our prior model of restoration.

\section{B. Unsupervised Markovian Segmentation}

To this end, the Markovian framework considers a couple of random fields $(Y, Z)$, with $Y=\left\{Y_{s}, s \in S\right\}$ the field of observations located on a lattice $S$ of $N$ sites $s$ (associated with the initial restoration estimated by Wiener filtering), and $Z=\left\{Z_{s}, s \in S\right\}$ the label field (related to the segmented image to be estimated). Each of the $Y_{s}$ takes its value in $\Lambda_{\text {obs }}=\{0, \ldots, 255\}$ (256 gray levels) and each $Z_{s}$ in a set of $K$ classes $\left\{e_{0}, e_{1}, \ldots, e_{K-1}\right\}$. In this notation, an upper case denotes a random field and a lower case, a particular realization of this random field. The distribution of $(Y, Z)$ is defined, firstly, by $P_{Z}(z)$, the distribution of $Z$ supposed to be stationary and Markovian, and secondly, by the site-wise conditional data likelihoods $P_{Y_{s} \mid Z_{s}}\left(y_{s} \mid z_{s}\right)$. If the data are assumed to be independent conditioned on the labeling process $Z$, one gets $P_{Y \mid Z}=\prod_{s \in S} P_{Y_{s} \mid Z_{s}}$. Data likelihood $P_{Y \mid Z}(y \mid z)$ depends on a parameter vector $\Phi$. Joint and posterior distributions $P_{Z, Y}(z, y)=P_{Z}(z) P_{Y \mid Z}(y \mid z)$ and $P_{Z \mid Y}(z \mid y) \propto P_{Z}(z) P_{Y \mid Z}(y \mid z)$, thus, depend on $\Phi$.

In the Markovian framework, image segmentation in $K$ classes can be viewed as a statistical labeling problem according to a global Bayesian formulation in which the posterior distribution $P_{Z \mid Y}(z \mid y) \propto \exp \{-U(z, y)\}$ has to be maximized [4]. This is the maximum a posteriori (MAP) estimation. In the standard case of the Potts prior model [4], the corresponding posterior energy to be minimized is

$$
U(z, y)=\sum_{s \in S} \Psi_{s}\left(z_{s}, y_{s}\right)+\sum_{\langle s, t\rangle} \beta_{s t}\left[1-\delta\left(z_{s}, z_{t}\right)\right]
$$

where $\Psi_{s}\left(z_{s}, y_{s}\right)=-\ln P_{Y_{s} \mid Z_{s}}\left(y_{s} \mid z_{s}\right), \delta$ is the delta

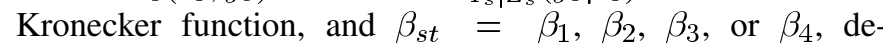
pending on whether the pair of neighboring sites (relative to the second-order neighborhood system), or clique, $\langle s, t\rangle$ is horizontal, vertical, right diagonal, or left diagonal [4]. In this energy setting, the first energy term expresses the adequacy between observations and labels, whereas the second one is related to the a priori.

In our application, we take a Gaussian law, $\mathcal{N}\left(\mu, \sigma^{2}\right)$ as degradation model to describe the luminance distribution within each class. To perform an unsupervised segmentation, we thus have to estimate the parameter vector $\Phi=\left[\left(\mu_{1}, \sigma_{1}\right), \ldots,\left(\mu_{K}, \sigma_{K}\right)\right]$ of the likelihood model, i.e., the parameters for each law associated to each class of the segmented image. To this end, we resort, to an iterative method of estimation called ICE [33]-[36] which gives the best estimation of $\hat{\Phi}$ in the least-squares sense and which can be viewed as a 


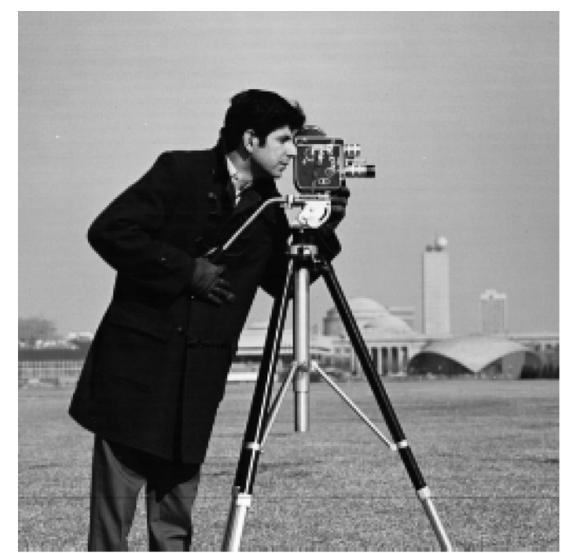

(a)

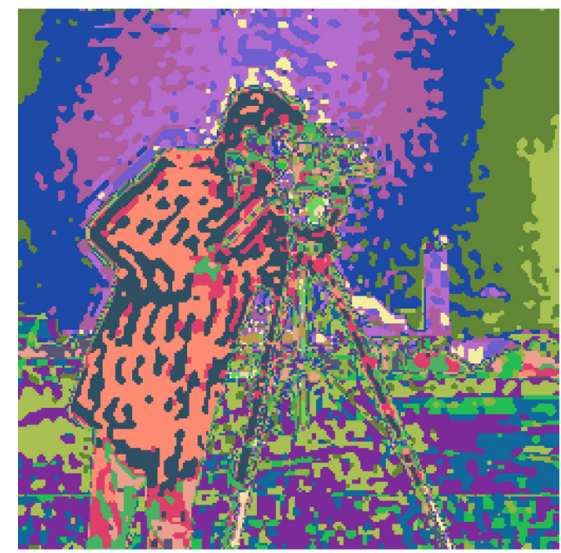

(d)

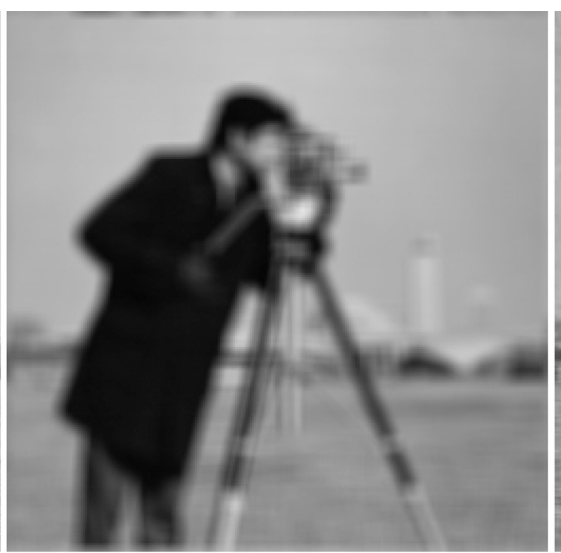

(b)

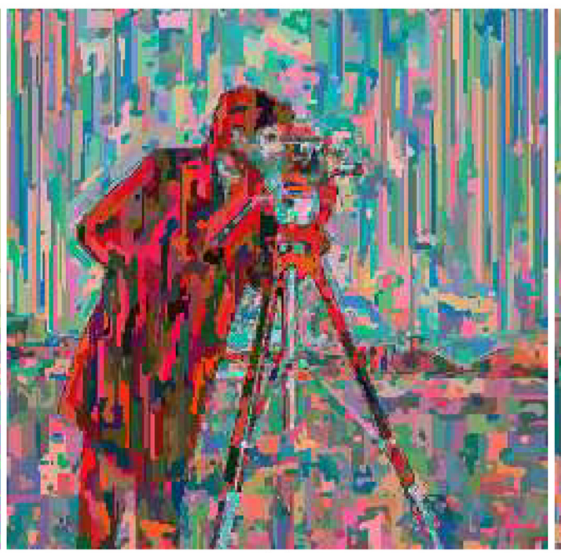

(e)

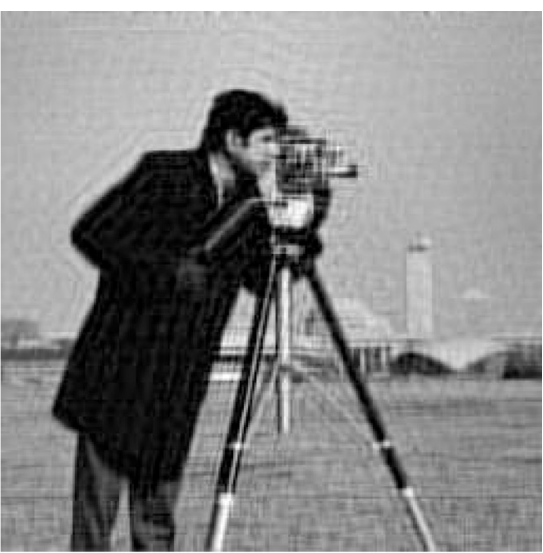

(c)

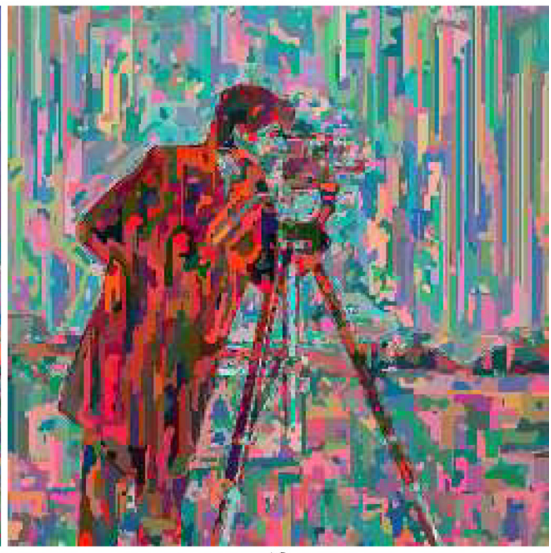

(f)

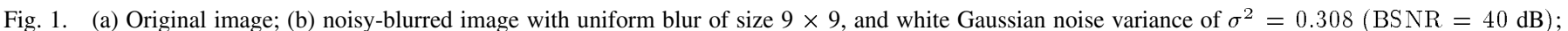

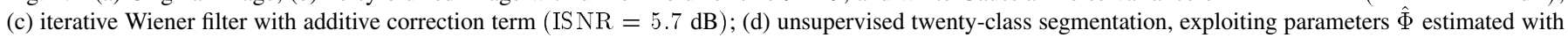

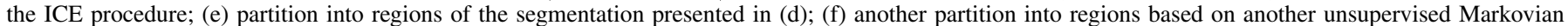
segmentation. (Color version available online at http://ieeexplore.ieee.org.)

stochastic and Markovian EM [36] procedure for the particular problem of the Gaussian distribution mixture estimation of the luminance within an image. This ICE procedure is initialized by a K-means clustering procedure [37] as proposed in [32]. In doing so, we assume as first approximation that the luminance distributions for each class are spherical with equal volumes (or equivalently, Gaussian distributions with identical variance). The obtained partitions by the K-means clustering procedure allow to obtain a rough estimation of the distribution mixture parameters (mean and variance of the Gaussian distribution for each class) which are then used to initialize the ICE estimation procedure.

Once $\hat{\Phi}$ is estimated, (12) is optimized using a classical iterative local update strategy called Iterated Conditional Modes (ICM) [4] algorithm. For the initialization of this iterative algorithm, we exploit the segmentation map obtained in a ML sense.

In our application, we take $\beta=\beta_{1}=\ldots=\beta_{4}=1$ for the parameters of the a priori model and $K=20$ classes. Fig. 1(d) displays an example of unsupervised twenty-class segmentation, exploiting parameters $\hat{\Phi}$ estimated with the ICE procedure.

\section{Partition Into Regions}

We now exploit this oversegmentation in order to get a reliable partition $\hat{\mathcal{R}}$ of the image into homogeneous regions. To this end, we simply search the set of disjoint regions (i.e., the set of connected pixels belonging to the same class). In order to limit regions with a large number of pixels which could produce an undesirable "staircase" or quantization effects by our regularization/prior term (which tends to favor piecewise smooth restorations), we subdivide all the regions with more than 100 pixel size.

Due to the stochastic aspect of the ICE estimation procedure, we can obtain, for several seeds, different segmentations and thus different partitions into regions of the same input image [see Fig. 1(e) and (f)]. These simulations of region maps are differently partitioned mostly on homogeneous regions without observable discontinuities (such as the sky region or the black coat of the cameraman). Nevertheless, the localized characteristics of the image such as the main structures, the edges and the details are preserved by these partitions which define a set of possible low-level representations of the image.

Fig. 1(d) and (e), respectively, shows the result of an unsupervised twenty-class segmentation on a Wiener filtering of the CAMERAMAN image and the partition into regions induced from this segmentation map. Fig. 1(e) and (f) displays two examples of region partition of the CAMERAMAN image given by our procedure. Fig. 1(e) shows 4834 regions with variable sizes (1 to 99 pixels) and an average size of 13.6 pixels size. 


\section{EXPERIMENTAL RESULTS}

In all the experiments, we have considered the gradient descent iterative procedure defined in (8) [with the use of (9) and with $\gamma=1$ ].

The initial estimate $\hat{x}^{[0]}$ for the iterative restoration procedure and used as observation for the segmentation step is given by the iterative Wiener filtering. The number of iteration of the ICE procedure is set to $20 .{ }^{1}$

The convergence criterion of the proposed restoration procedure is the stability of the MAP energy, i.e.

$$
\frac{E\left(\hat{x}^{[n]}\right)-E\left(\hat{x}^{[n-1]}\right)}{E\left(\hat{x}^{[n]}\right)} \leq \delta
$$

with $\delta$ is a threshold, typically set, in our application, to $10^{-5} \sigma^{2}$. The regularization parameter $\alpha$, that controls the contribution of the likelihood and prior terms is given by

$$
\alpha=\zeta \cdot \frac{\left\|y-h * \hat{x}^{[0]}\right\|^{2}}{\Omega\left(\hat{x}^{[0]}\right)}
$$

where $\hat{x}^{[0]}$ is the first restoration result given by the iterative Wiener Filter presented in Section III-A. This expression is inspired by the work of Katsaggelos et al. in [38] and will allow to make our approach less sensitive to the regularization parameter $\alpha$ for a fixed value of $\zeta$.

\section{A. Sensitivity to Parameters}

First, we have tested the influence of the four following parameters, namely, $1-\beta\left(=\beta_{1}=\ldots=\beta_{4}\right)$, the regularization term of the segmentation step, $2-K$, the number of classes of the segmentation step, $3-N_{\text {Seg }}$, the number of partitions into regions and, $4-\zeta$, the regularization factor used in the restoration step [see (14)], on the result of the SNR improvement measure. In this first set of experimentations, we consider the following degradation model on the CAMERAMAN image; a uniform blur of size $7 \times 7$ and a noise variance $\sigma^{2}=2$ (corresponding to BSNR $=32 \mathrm{~dB}$ ). Fig. 4 shows the evolution of the ISNR improvement along several discrete values of these parameters. Let us note the following.

- For $K=1$, i.e., one class for the segmentation map and $N_{\text {Seg }}=1$ (number of segmentations), the proposed model is analogous to the simplest prior model used in the regularization framework, i.e., $\Omega(x)=\|x\|^{2}$ which yields to the so-called Tikhonov regularization with the identity [6], or equivalently to the inverse Wiener filter.

- For $\beta=0$, the estimation of the segmentation map is done in the ML sense and the estimation procedure, used to estimate the likelihood distribution of this ML segmentation, is analogous to the SEM procedure [36].

- For $\zeta=0$, i.e., $\alpha=0$, the proposed iterative procedure is analogous to the Landweber iteration [39].

\footnotetext{
${ }^{1}$ We can also use an adaptive criterion for this number of iterations as the one proposed in [32] which is based on an indicator of the "stability" of the procedure. When this one falls below a given threshold, the sequence of $\Phi^{[k]}$ is assumed to have reached an equilibrium and the procedure is ended. Nevertheless, experimental results have shown that 15 or 20 iterations were sufficient for the convergence of the ICE procedure for all the experiments reported in this paper.
}

TABLE I

BLUR, NOISE VARIANCE, AND BSNR (dB) FOR THE FOUR EXPERIMENTS

\begin{tabular}{l||l||l||l}
\hline \hline & Blur & $\sigma^{2}$ & BSNR \\
\hline \hline Exp1 & $9 \times 9$ uniform & $\approx 0.308$ & 40 \\
\hline Exp2 & $h_{i j}=\left(1+i^{2}+j^{2}\right)^{-1}, i, j=-7, \ldots, 7$ & 2 & 32 \\
\hline Exp3 & $h_{i j}=\left(1+i^{2}+j^{2}\right)^{-1}, i, j=-7, \ldots, 7$ & 8 & 26 \\
\hline Exp4 & {$[1,4,6,4,1]^{t}[1,4,6,4,1] / 256$} & 49 & 18 \\
\hline \hline
\end{tabular}

TABLE II

ISNR (dB) OF THE Proposed Algorithm AND Methods [16], [18], [19], [26], [30], [40] FOR THE FOUR EXPERIMENTS

\begin{tabular}{l||cccc}
\hline \hline \multicolumn{1}{l||}{} & \multicolumn{4}{c}{ ISNR (dB) } \\
\hline Method & Exp1 & Exp2 & Exp3 & Exp4 \\
\hline \hline Mig-3 & 8.71 & 7.58 & 5.75 & 1.60 \\
Mig-2 & 8.42 & 8.03 & 5.72 & 1.78 \\
Mig-1 & 8.23 & 7.58 & 5.70 & 1.63 \\
\hline Bioucas-Dias [19] & 8.10 & 7.40 & 5.15 & 2.85 \\
Figueiredo \&. Nowak [18] & 7.59 & 6.93 & 4.88 & 2.94 \\
Neelamani et al. [26] & 7.30 & - & - & - \\
Banham \& katsaggelos [30] & 6.70 & - & - & - \\
Jalobeanu et al. [16] & - & 6.75 & 4.85 & - \\
Liu \& Moulin [40] & - & - & - & 1.08 \\
\hline \hline
\end{tabular}

TABLE III

DEGRADATION MODEL EXP5 AND OBTAINED ISNR

\begin{tabular}{l||l||ll}
\hline \hline Image name & Exp5 & ISNR & \\
\hline \hline Cameraman & Blur, $\sigma^{2}$, BSNR: & Mig-3 & $3.50 \mathrm{~dB}$ \\
& $5 \times 5$ uniform & Mig-2 & $3.73 \mathrm{~dB}$ \\
& $\sigma^{2}=33.3$ & Mig-1 & $3.50 \mathrm{~dB}$ \\
& BSNR $=20 \mathrm{~dB}$ & {$[42]$} & $3.43 \mathrm{~dB}$ \\
\hline \hline
\end{tabular}

Experiments show that the proposed restoration model is not too sensitive to parameters $\beta, K$ and $N_{\mathrm{Seg}}$ if $\beta \in[0.5,1.5]$, $K \in[15,30]$ and $N_{\text {Seg }}>1$. In fact, experiments have shown that this last parameter has to be all the more greater than 1 that the variance of the noise or the degradation of the initial Wiener filtering restoration solution is important. On the other hand, the proposed method seems a bit sensitive to the regularization parameter $\zeta$.

For $\left(\beta=1.0, K=20, N_{\text {Seg }}=5, \zeta=0.1\right)$, we obtain an ISNR $=6.71 \mathrm{~dB}$ for the CAMERAMAN image and for the above-mentioned degradation model (uniform blur $7 \times 7$ and $\sigma^{2}=2$ ).

\section{B. Comparison With State-of-the-Art Methods}

We now present a set of experimental results and comparisons illustrating the performance of the proposed approach. We have taken for all the following experiments $\beta=1.0$ and $K=20$ classes for the segmentation step and $N_{\mathrm{Seg}}=5$ partitions into regions and finally $\zeta=0.2$ for the restoration step.

For the first four experiments, we have replicated the scenarios used in the evaluation of state-of-the-art methods described in [16], [18], [19], [26], [30], [40], with which we compare the proposed approach. In these experiments, original images are CAMERAMAN (experiments 1, 2, and 3) and LENA 


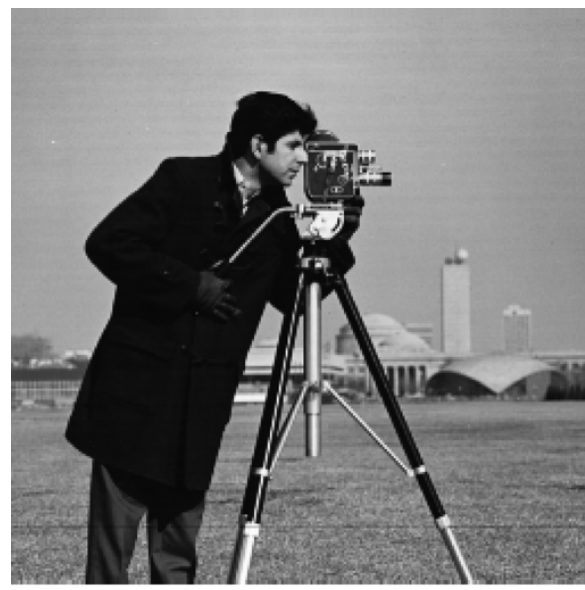

(a)

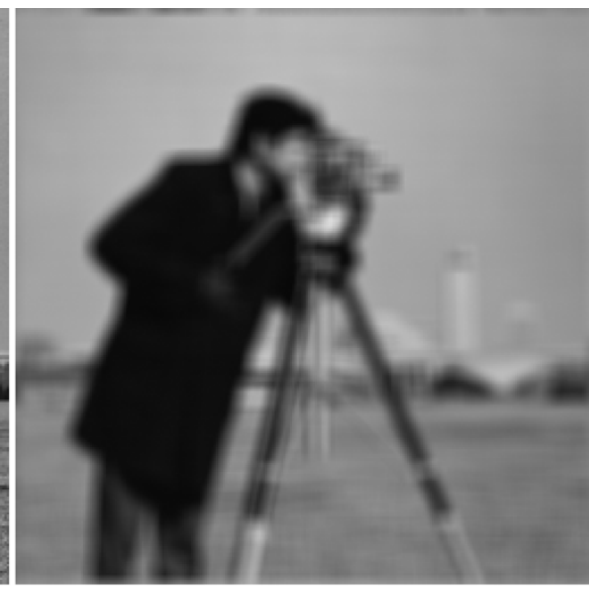

(b)

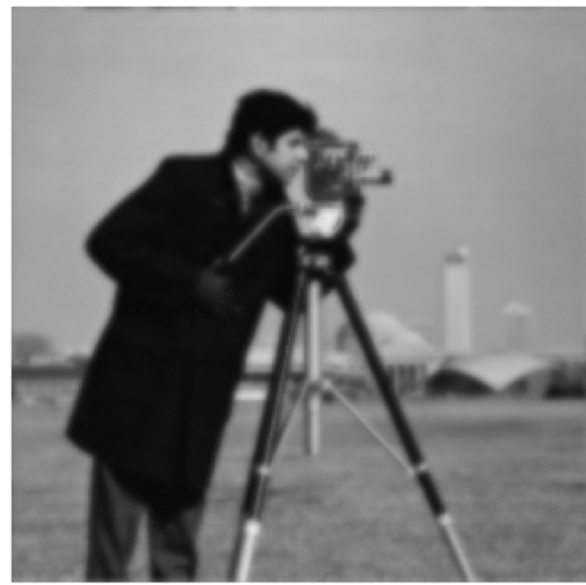

(d)

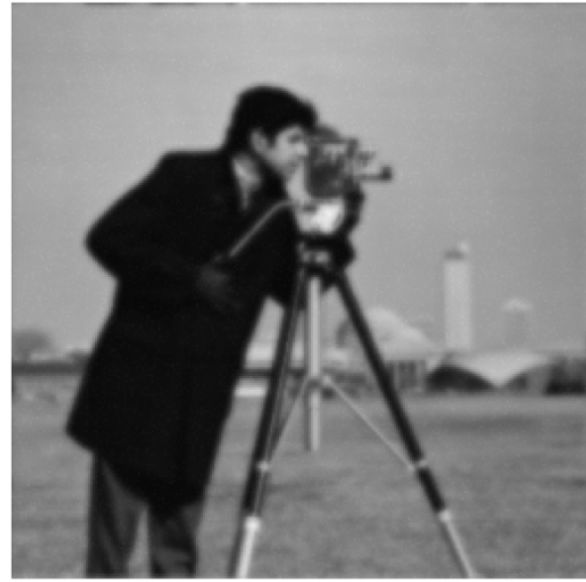

(f)

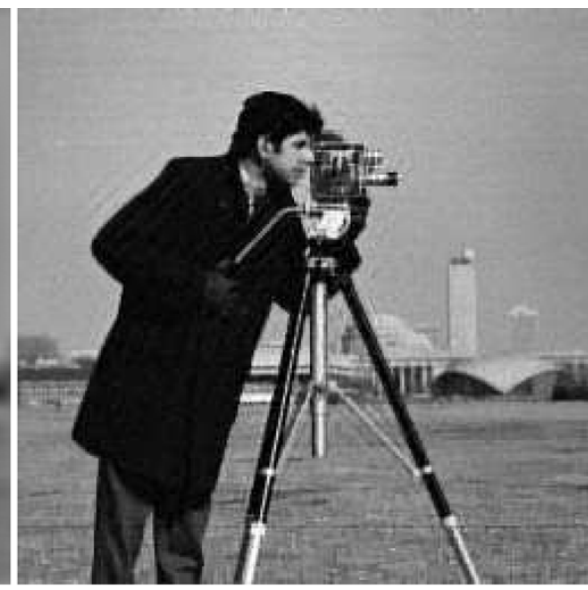

(c)

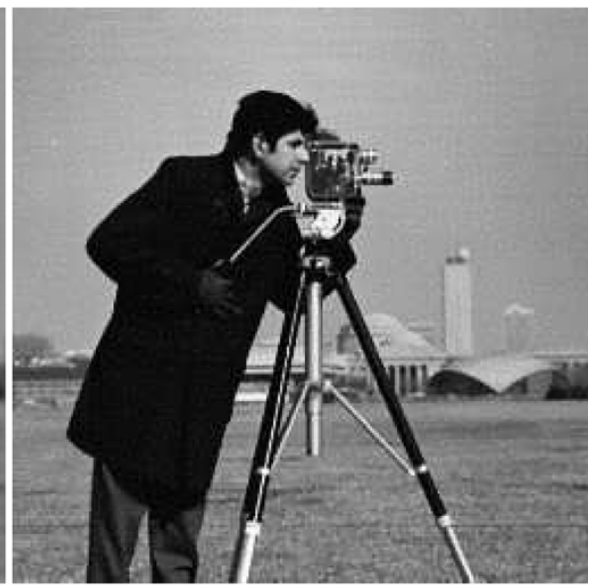

(e)

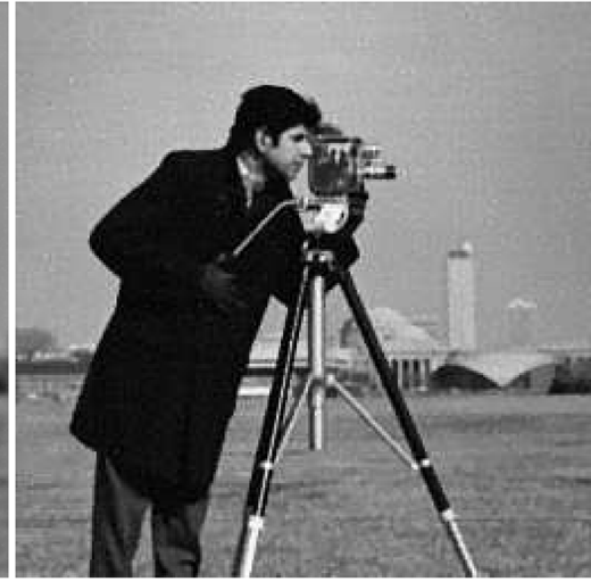

(g)

Fig. 2. (a) Original image. (b), (d), (f) Noisy-blurred image for respectively Exp1, Exp2, and Exp3 (see Table I). (c), (e), (g) Restored image using the proposed approach [algorithm Mig-3 for (c) and Mig-1 for (e) and (g)] (see Table II).

(experiment 4 ) both of size $256 \times 256$. Table I displays the blur, the noise and the resulting BSNR (the ratio between the variance of the noise and the variance of blurred image without noise) for each of the four experiments. We have considered three variants of our algorithm.

- First, we consider one pass (i.e., segmentation-restoration) of our algorithm with the parameter vector $(\alpha, \delta)$ estimated respectively by (14) and (13) (algorithm called Mig-1).
- Second, one pass of our algorithm with the parameter vector $(\alpha, \delta)$ manually fixed with supervised values (algorithm called Mig-2).

- Third, two passes of the algorithm. More precisely, the final result of restoration is used to re-estimate both a better segmentation and then to refine, in a second pass our prior model, and also to re-estimate the initial Wiener filtering estimate given to the second pass of the iterative restoration process (8) (via the Fourier spectrum of $x$ 


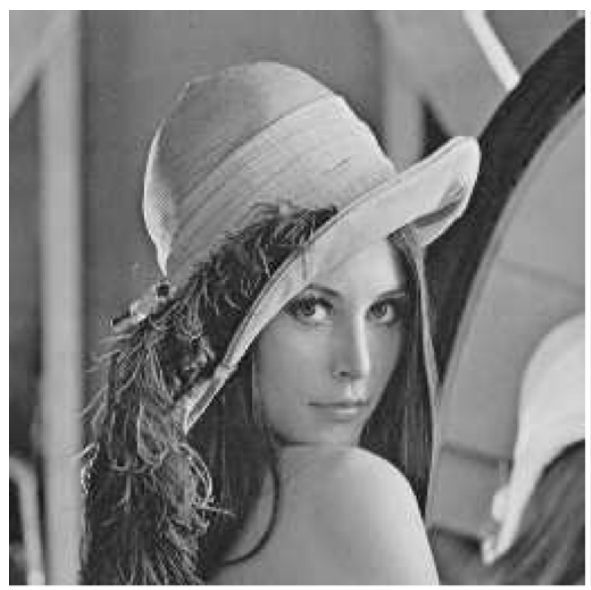

(a)

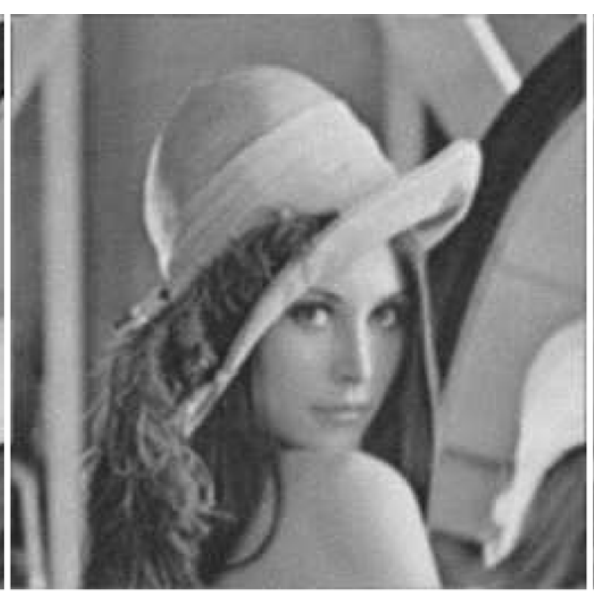

(b)

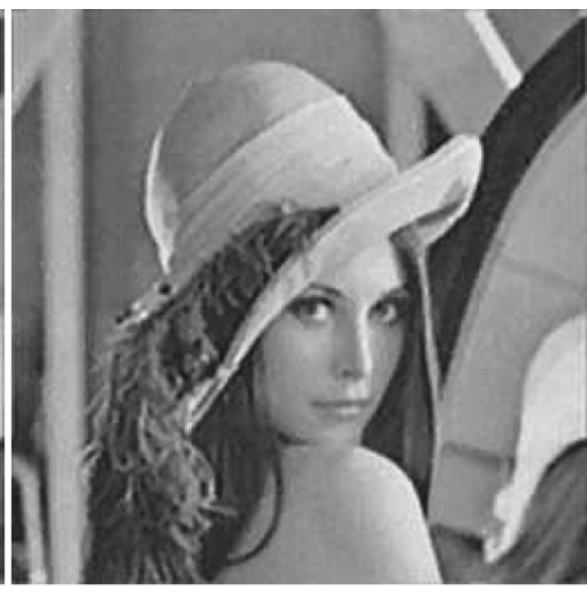

(c)

Fig. 3. (a) Original image. (b) Noisy-blurred image for Exp4 (see Table I). (c) Restored image using the proposed approach (algorithm Mig-1) (see Table II).

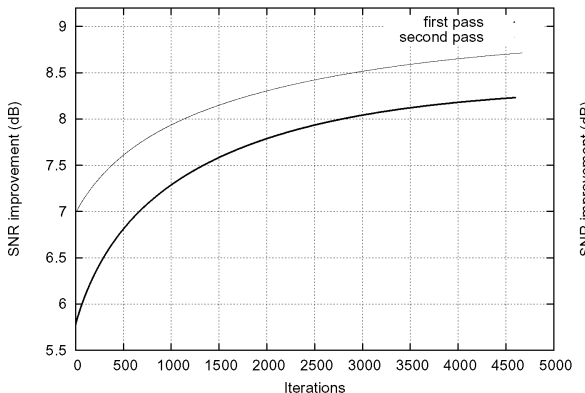

(a)

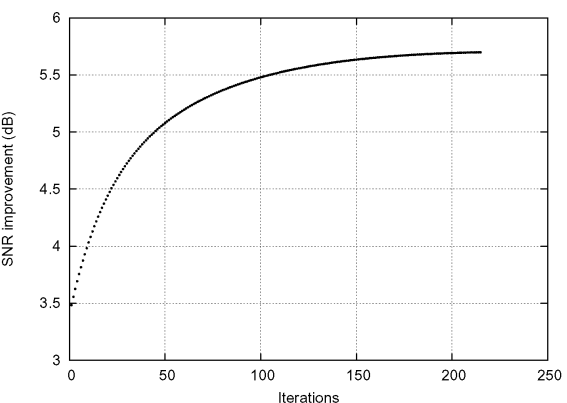

(b)

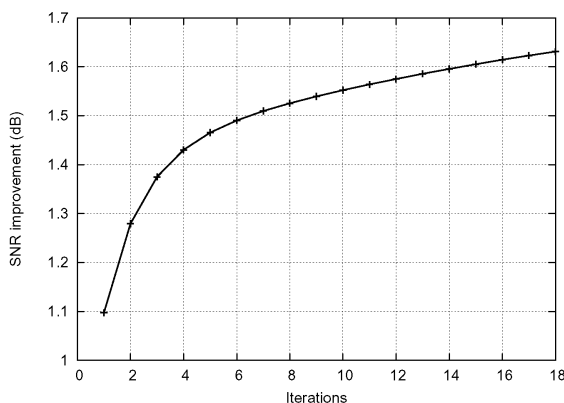

(c)

Fig. 4. Evolution of the SNR improvement along the iteration of the gradient descent process for (see Table I) (a) Exp1, during the first and second pass of the algorithm (Mig-3), (b) Exp3 (algorithm Mig-1), (c) Exp4 (algorithm Mig-1).

which is now estimated by the first final result of restoration). The parameter vector $(\alpha, \delta)$ is estimated respectively by (14) and (13) (algorithm called Mig-3).

Table II shows the obtained signal-to-noise improvements (ISNR) obtained by the three versions of our approach compared to the algorithms described in [16], [18], [19], [26], [30], and [40] for the four experiments. Fig. 2 and 3 show, respectively, the original image, the blurred noisy image and the restored image using the proposed approach. Fig. 4 shows the evolution of the ISNR improvement along the iteration of the gradient descent process for the example of the four degradation models described in Table I.

Our approach performs competitively, in several cases better than the best existing methods in benchmark tests, except for Exp4 which is not far from a pure denoising problem for which wavelet-based restoration such as [18], [19] give better ISNR results. Besides, the interest of doing several partitions into regions, in order to minimize the effects of the dependence of a bad segmentation map on the restoration result, can be noticed when we compare these ISNR results to the ones given when $K=1$ in [41] (a short and preliminary version of this work).

We have also replicated the degradation model described in [42] for which we indicate the ISNR obtained with the three variants of the proposed approach [see Table IV(b)]. We have also compared our model on the degradation model described by Molina et al. in [43] in which two edge-preserving methods was implemented and tested; respectively. 1) First, the use of a compound Gauss-Markov random fields (CGMRF), using an Ising model to represent the upper level and a line process to model the abrupt transitions (and acting as an activator or inhibitor of the relation between two neighbor pixels). In this model, the solution is estimated thanks to an extension of the classical simulated annealing. 2) Second, the ARTUR model of [44] also implemented and tested in [43] in the restoration context. The degradation model consists of a Gaussian noise with variance $\sigma^{2}=62.5$ added to the CAMERAMAN image blurred by an atmospherical PSF of the form $\propto\left[1+\left(i^{2}+j^{2}\right) / 16\right]^{-3}$. In order to compare the quality of the restoration result, we use as in [43] the peak signal-to-noise ratio (PSNR) that, for two images $\hat{x}$ and $y$ of $N$ sites, is defined, in decibels, as $10 \log _{10}\left(\left[N \times 255^{2}\right] /\left[\|y-\hat{x}\|^{2}\right]\right)$ and we summarize the results in Table IV. Once again, we can noticed that our approach performs competitively.

\section{Discussion}

We can notice that the proposed algorithm is especially efficient for the deblurring or deconvolution problem compared to the wavelet-based restoration methods which remain more efficient for, low SNR, noise-corrupted images. This can be seen on Fig. 6 which shows the evolution of the ISNR for a degradation model combining a $9 \times 9$ uniform blur and a BSNR varying from 40 to $5 \mathrm{~dB}$ and for our method versus a recent 


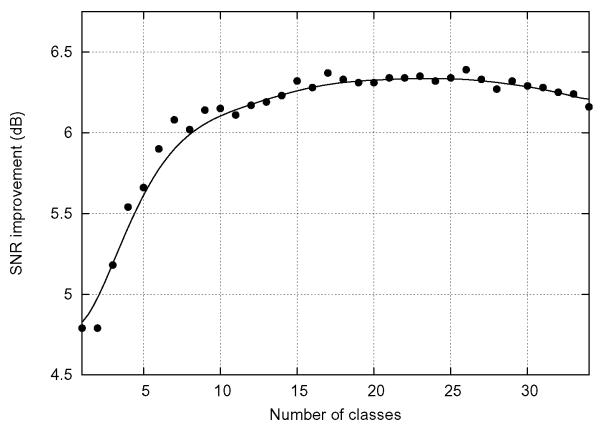

(a)

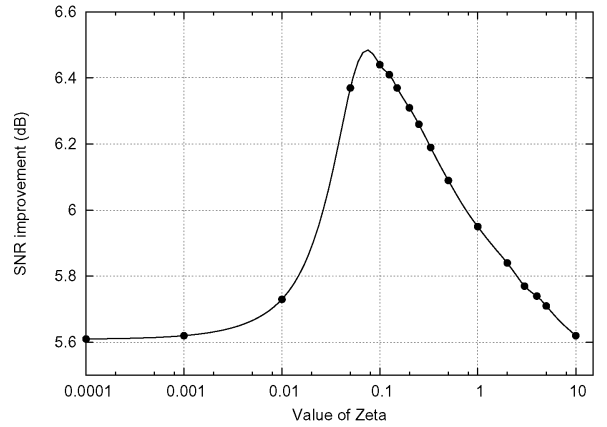

(c)

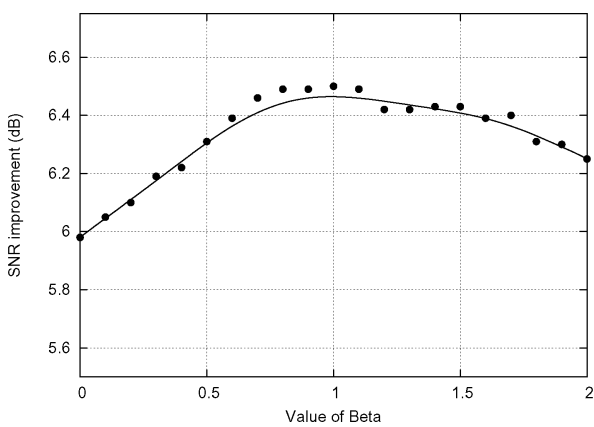

(b)

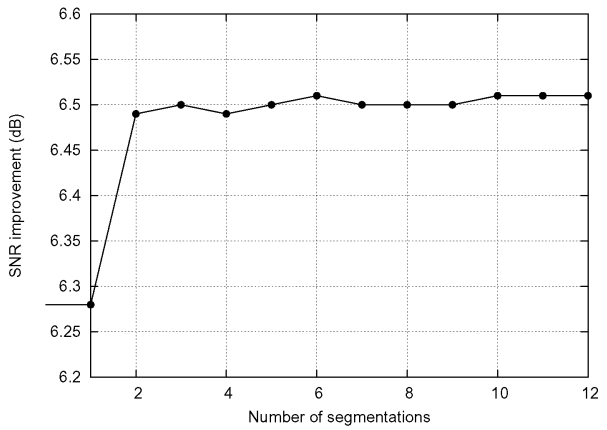

(d)

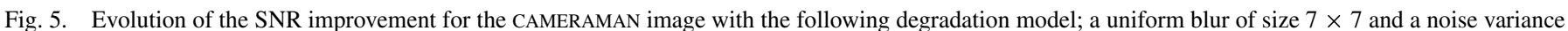

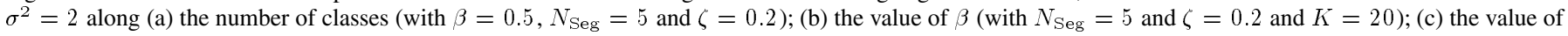
$\zeta$, (with $N_{\text {Seg }}=5, K=20$ and $\beta=0.5$ ); (d) the value of $N_{\text {Seg }}$ (with $\beta=0.5, \zeta=0.2$ and $K=20$ ).

TABLE IV

DEGRADATION MODEL EXP6 AND OBTAINED ISNR-PSNR

\begin{tabular}{l||l||ll}
\hline \hline Image name & Exp6 & ISNR - PSNR \\
\hline \hline Cameraman & Blur, $\sigma^{2}$, BSNR : & Mig-1 $\begin{array}{c}\text { PSNR=23.3 dB } \\
\text { (ISNR }=1.9 \mathrm{~dB})\end{array}$ \\
& & \\
& $\propto\left[1+\left(i^{2}+j^{2}\right) / 16\right]^{-3}$ & \\
& $\sigma^{2}=62.5$ & CGMRF [43] PSNR $=21.1 \mathrm{~dB}$ \\
& BSNR $=14 \mathrm{~dB}$ & ARTUR [44] PSNR $=20.8 \mathrm{~dB}$ \\
\hline \hline
\end{tabular}

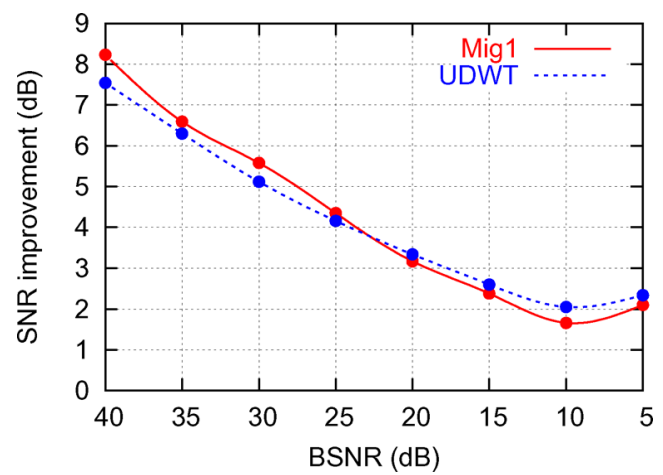

Fig. 6. Evolution of the SNR improvement for the CAMERAMAN image with the following degradation model; a uniform blur of size $9 \times 9$ and a BSNR varying from 40 to $5 \mathrm{~dB}$ (set of noise variances [0.308: 0.973: 3.080: 9.750: 30.80: 97.50: 308.50: 975]), for our method versus the UDWT-based restoration method proposed in [18]. (Color version available online at http://ieeexplore.ieee.org.)

wavelet-based restoration procedure. For this experiment, we use the restoration scheme proposed in [18] with the translation-

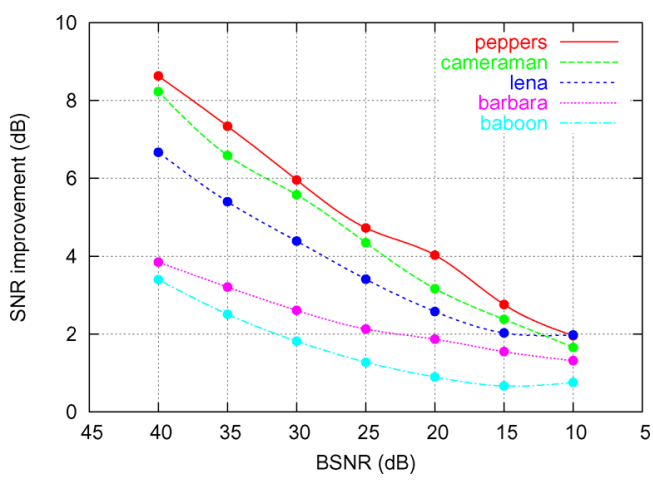

Fig. 7. Evolution of the SNR improvement versus the BSNR (for a uniform $9 \times 9$ blur and increasing noise variances) for respectively the following set of images with increasing texture complexity and activity, i.e., PEPPERS, CAMERAMAN, LENA, BARBARA, and BABOON images. (Color version available online at http://ieeexplore.ieee.org.)

invariant wavelet-based denoising rule proposed by the authors [using the undecimated discrete wavelet transform (UDWT)].

We have also noticed that the proposed deconvolution algorithm is also especially well suited for the class of piecewise smooth (or constant) images such as PEPPERS, ZELDA, or BOAT and less appropriated for highly textured image such as BABOON or BARBARA images. This can be seen on Fig. 7, which shows the evolution of the ISNR versus the BSNR for some well-known $256 \times 256$ images exhibiting an increasing texture complexity and activity.

In our setting, the use of (14) make the proposed approach relatively insensitive to the regularization parameter $\alpha$ for a fixed value of $\zeta$ (i.e., for algorithms Mig-1 and Mig-3). 
TABLE V

TIME IN SECONDS AND ITERATION NUMBER FOR THE FIVE EXPERIMENTS AND FOR ALGORITHM Mig-1

\begin{tabular}{l|l|l}
\hline \hline & Time $(\mathrm{sec})$ & Iteration \\
\hline \hline Exp1 & 1572 & 4599 \\
Exp2 & 522 & 690 \\
Exp3 & 258 & 215 \\
Exp4 & 125 & 18 \\
Exp5 & 110 & 43 \\
\hline \hline
\end{tabular}

We can also notice that the strategy of restoration called Mig-3 is not always ensured to improve the restoration results compared to Mig-1.

We finally can observe that for larger noise variance, convergence is achieved in fewer iterations. Table $\mathrm{V}$ shows the time in seconds and the number of iterations that each restoration took for each one of the four degradation models described in Table I and for algorithm Mig-1 summarized in Algorithm 1.

\section{CONCLUSION}

In this paper, we have proposed an adaptive edge-preserving Tikhonov regularization term for image restoration. This regularization term whose goal is to promote piecewise smooth over pre-estimated homogeneous regions are based on an unsupervised Markovian segmentation.

In this way, the proposed restoration is performed by using a two-level MRF model. The first one, used in the segmentation procedure, includes a low-level prior model namely, a simple Potts prior model ensuring homogeneity of the segmentation map and a likelihood model expressing the luminance distribution of each homogeneous region of the image. While the second exploits the segmentation result (via a partition into regions map) as a meta parameter of the adaptive prior model of restoration. This prior model efficiently expresses the expected local smoothness property in the image to be recovered. The restoration procedure finds the best solution that is consistent with the data that respects this local smoothness property. These two estimation problems (segmentation and restoration) are defined as the search of the MAP or equivalently as an optimization problem in which the posterior energy has to be minimized. To this end, we use a gradient-based algorithm for the segmentation and restoration step.

Let us note that the proposed regularization strategy is, in fact, the generalization for $K$ (number of classes) $\neq 1$ and $N_{\text {Seg }}=$ 1 (number of segmentations) of the so-called Tikhonov regularization with the identity [6]. In our approach, this Tikhonov constraint with the identity is locally applied to pre-estimated homogeneous regions. Let us note that this adaptive segmentation-based regularization strategy could be easily tested for the other global regularization terms proposed by the image processing community (see Section I-B).

The proposed technique efficiently adapts to local characteristics of the data and allows to obtain results which simultaneously exhibit no ringing or blocky artifacts, sharp edges, correctly restored textures and very low noise in homogeneous areas. In comparison with state-of-the-art methods, the experiments reported in this paper demonstrate that the discussed

\section{Segmentation-Based Deconvolution Algorithm (Mig-1)}

$N_{\text {seg }} \quad$ Number of segmentations/partitions

$K \quad$ Number of classes of the segmentation

$\sigma^{2} \quad$ Variance of the noise

\section{1. $\hat{x}^{[0]} \leftarrow$ Iterative Wiener Filtering of $y$}

\section{Unsupervised Segmentations/Partitions}

for $k=1$ to $N_{\text {Seg }}$ do

- K-means clustering of each pixel of $\hat{x}^{[0]}$ into $K$ clusters, initially randomly chosen $(\operatorname{SEED}=k)$

- $\Phi^{[0]} \leftarrow$ ML fitting of Gaussian law for each cluster

- $z_{\mathrm{ML}}^{[0]} \leftarrow$ ML segmentation of $\hat{x}^{[0]}$ based on $\Phi^{[0]}$

- $\Phi \leftarrow$ ICE algorithm on $\hat{x}^{[0]}$ (initialized with $z_{\mathrm{ML}}^{[0]}$ and $\operatorname{SEED}=k$ )

- $z_{\mathrm{ML}} \leftarrow$ ML segmentation of $\hat{x}^{[0]}$ based on $\Phi$

- $z_{\mathrm{MAP}} \leftarrow$ ICM segmentation (initialized with $z_{\mathrm{ML}}$ ) and based on $P_{Z \mid X, \Phi}\left(. \mid \hat{x}^{[0]}, \Phi\right)$

- $z_{k} \leftarrow$ Partition into regions (of less than 100 pixel size) of $z_{\mathrm{MAP}}$

$\alpha \leftarrow \zeta \frac{\left\|y-h * \hat{x}^{[0]}\right\|^{2}}{\Omega\left(\hat{x}^{[0]}\right)}$

\section{Segmentation-Based Deconvolution}

$$
\begin{aligned}
& \text { while }\left[E\left(\hat{x}^{[n+1]}\right)-E\left(\hat{x}^{[n]}\right)\right] / E\left(\hat{x}^{[n+1]}\right) \leq 10^{-5} \sigma^{2} \\
& \text { do } \\
& \qquad \begin{aligned}
\hat{x}^{[n+1]} & \leftarrow \hat{x}^{[n]}+\left(h^{\#} *\left(y-h * \hat{x}^{[n]}\right)\right. \\
& \left.-\alpha \rho^{\prime}\left(\hat{x}^{[n]}\right)\left[\hat{x}^{[n]}-\frac{1}{N_{\text {Seg }}} \sum_{k=1}^{N_{\text {Seg }}} \Gamma_{k}\left(\hat{x}^{[n]}\right)\right]\right)
\end{aligned}
\end{aligned}
$$

- Compute the MAP

$$
\begin{aligned}
& E\left(\hat{x}^{[n+1]}\right) \leftarrow\left\{\left\|y-h * \hat{x}^{[n+1]}\right\|^{2}\right. \\
& \left.+\frac{\alpha}{2}\left\|\hat{x}^{[n+1]}-\frac{1}{N_{\text {Seg }}} \sum_{k=1}^{N_{\text {Seg }}} \Gamma_{k}\left(\hat{x}^{[n+1]}\right)\right\|^{2}\right\}
\end{aligned}
$$

$n \leftarrow n+1$

Algorithm. 1. Segmentation-based deconvolution algorithm (Mig-1).

method performs competitively, and often better, than the best existing ones in benchmark tests.

In addition, the Gaussian likelihood model used in the segmentation process is the simplest one. The result could be improved by using more accurate modeling. Besides, the joint estimation of segmentation and restoration could be tested. Nevertheless, it is not sure that the increase in both the dimensionality of the required optimization and the complexity of the parameter estimation procedure would allow to improve the speed and the accuracy of the restoration result.

Finally, this adaptive regularization term can be efficiently applied in order to regularize many types of inverse problems in image processing or computer vision such as tomography, motion estimation, stereovision, superresolution, etc. 


\section{ACKNOWLEDGMENT}

The author would like to thank the reviewers for their many valuable comments and suggestions that helped to improve this paper.

\section{REFERENCES}

[1] R. Molina, "On the hierarchical Bayesian approach to image restoration: applications to astronomical images," IEEE Trans. Pattern Anal. Mach. Intell., vol. 16, no. 11, pp. 1122-1128, Nov. 1994.

[2] J. Hadamard, Lectures on Cauchy's Problem in Linear Partial Differential Equation. New Haven, CT: Yale Univ. Press, 1923.

[3] S. Geman and D. Geman, "Stochastic relaxation, Gibbs distributions and the Bayesian restoration of images," IEEE Trans. Pattern Anal. Mach. Intell., vol. 6, no. 6, pp. 721-741, Jun. 1984.

[4] J. Besag, "On the statistical analysis of dirty pictures," J. Roy. Statist. Soc., vol. B-48, pp. 259-302, 1986.

[5] A. N. Tikhonov and V. Arsenin, Solutions of Ill-posed Problems. New York: Winston and Sons, 1977.

[6] A. N. Tichonov, "Regularization of incorrectly posed problems," Soviet Math. Dokl, vol. 4, pp. 1624-1627, 1963.

[7] G. Wang, J. Zhang, and G.-W. Pan, "Solution of inverse problems in image processing by wavelet expansion," IEEE Trans. Image Process., vol. 4, no. 5, pp. 579-593, May 1995.

[8] R. Molina, A. K. Katsaggelos, and J. Abad, "Bayesian image restoration using a wavelet-based subband decomposition," presented at the IEEE Int. Conf. Acoust., Speech, Signal Processing, Mar. 1999.

[9] B. Ripley, Spatial Statistics. New York: Wiley, 1981.

[10] A. R. Barron, Non-Parametric Function Estimation and Related Topics, G. Roussas, Ed. Dordrecht, The Netherlands: Kluwer, 1991, pp. 561-576.

[11] J. Liu and P. Moulin, "Complexity-regularized image denoising," IEEE Trans. Image Process., vol. 10, no. 6, pp. 841-851, Jun. 2001.

[12] P. Moulin and J. Liu, "Analysis of multiresolution image denoising schemes using generalized Gaussian and complexity priors," IEEE Trans. Inf. Theory, vol. 45, no. 3, pp. 909-919, May 1999.

[13] J. Rissanen, Stochastic Complexity in Statistical Inquiry, Singapore: World Scientific, 1989.

[14] M. Belge, M. E. Kilmer, and E. L. Miller, "Wavelet domain image restoration with adaptive edge-preserving regularization," IEEE Trans. Image Process., vol. 9, no. 4, pp. 597-608, Apr. 2000.

[15] Y. Wan and R. D. Nowak, "A Bayesian multiscale approach to joint image restoration and edge detection," in Proc. SPIE Wavelet Applications in Signal and Image Processing VII, vol. 3813, Jul. 1999, pp. 73-84.

[16] A. Jalobeaunu, N. Kingsbury, and J. Zerubia, "Image deconvolution using hidden Markov tree modeling of complex wavelet packets," presented at the IEEE Int. Conf. Image Processing, Oct. 2001.

[17] A. Jalobeaunu, L. Blanc-Feraud, and J. Zerubia, "Satellite image deblurring using complex wavelet packets," Int. J. Comput. Vis., vol. 51, no. 3, pp. 205-217, 2003

[18] M. A. T. Figueiredo and R. D. Nowak, "An EM algorithm for waveletbased image restoration," IEEE Trans. Image Process., vol. 12, no. 8, pp. 906-916, Aug. 2003.

[19] J. Bioucas-Dias, "Bayesian wavelet-based image deconvolution: A GEM algorithm exploiting a class of heavy-tailed priors," IEEE Trans. Image Process., vol. 15, no. 4, pp. 937-951, Apr. 2006.

[20] A. P. Dempster, N. M. Laird, and D. B. Rubin, "Maximum likelihood from incomplete data via the EM algorithm," J. Roy. Statist. Soc., pp. 1-38, 1976.

[21] R. Acar and C. R. Vogel, "Analysis of total variation penalty methods," Inv. Probl., vol. 10, pp. 1217-1229, 1994.

[22] D. C. Dobson and F. Santosa, "Recovery of blocky images from noisy and blurred data," SIAM J. Appl. Math., vol. 56, pp. 1181-1198, 1996.

[23] D. Geman and G. Reynolds, "Constrained image restoration and the recovery of discontinuities," IEEE Trans. Pattern Anal. Mach. Intell., vol. 14, no. 3, pp. 367-383, Mar. 1992.

[24] D. Geman and C. Yang, "Nonlinear image recovery with half-quadratic regularization," IEEE Trans. Image Process., vol. 4, no. 7, pp. 932-945, Jul. 1995.

[25] P. Charbonnier, L. Blanc-Feraud, G. Aubert, and M. Barlaud, "Deterministic edge-preserving regularization in computed imaging," IEEE Trans. Image Process., vol. 5, no. 12, pp. 298-311, Dec. 1997.
[26] R. Neelamani, H. Choi, and R. Baraniuk, "Forward: Fourier-wavelet regularized deconvolution for ill-conditioned systems," IEEE Trans. Signal Process., vol. 52, no. 2, pp. 418-433, Feb. 2004.

[27] J. Woods, S. Dravida, and R. Mediavilla, "Image estimation using double stochastic Gaussian random field models," IEEE Trans. Pattern Anal. Mach. Intell., vol. 9, pp. 245-253, 1987.

[28] C. A. Bouman and K. D. Sauer, "A generalized Gaussian image model for edge-preserving MAP estimation," IEEE Trans. Image Process., vol. 2, no. 3, pp. 296-310, Mar. 1993.

[29] A. Jalobeaunu, L. Blanc-Feraud, and J. Zerubia, "An adaptive Gaussian model for satellite image deblurring," IEEE Trans. Image Process., vol. 13, no. 4, pp. 613-621, Apr. 2004.

[30] M. R. Banham and A. K. Katsaggelos, "Spatially adaptive wavelet-based multiscale image restoration," IEEE Trans. Image Process., vol. 5, no. 4, pp. 619-634, Apr. 1996.

[31] A. D. Hillery and R. T. Chin, "Iterative Wiener filters for image restoration,” IEEE Trans. Signal Process., vol. 39, no. 8, pp. 1892-1899, Aug. 1991.

[32] M. Mignotte, C. Collet, P. Pérez, and P. Bouthemy, "Sonar image segmentation using a hierarchical MRF model," IEEE Trans. Image Process., vol. 9, no. 7, pp. 1216-1231, Jul. 2000.

[33] F. Salzenstein and W. Pieczynski, "Parameter estimation in hidden fuzzy Markov random fields and image segmentation," Graph. Models Image Process., vol. 59, no. 4, pp. 205-220, 1997.

[34] W. Pieczynski, "Champs de Markov cachés et estimation conditionnelle itérative," Revue Traitement Du Signal, vol. 11, no. 2, pp. 141-153, 1994.

[35] B. Braathen, P. Masson, and W. Pieczynski, "Global and local methods of unsupervised Bayesian segmentation of images," Graph. Vis., vol. 2, no. 1 , pp. 39-52, 1993.

[36] P. Masson and W. Pieczynski, "SEM algorithm and unsupervised statistical segmentation of satellite images," IEEE Trans. Geosci. Remote Sens., vol. 31, no. 3, pp. 618-633, Mar. 1993.

[37] S. Banks, Signal Processing, Image Processing and Pattern Recognition. Englewood Cliffs, NJ: Prentice-Hall, 1990.

[38] A. K. Katsaggelos and M. G. Kang, "Iterative evaluation of the regularization parameter in regularized image restoration," J. Vis. Commun. Image Represent., vol. 3, no. 4, pp. 446-455, 1992.

[39] L. Landweber, "An iterative formula for fredholm integral equations of the first kind," Amer. J. Math., vol. 73, pp. 615-624, 1951.

[40] J. Liu and P. Moulin, "Complexity-regularized image restoration," in Proc. IEEE Int. Conf. Image Processing, Oct. 1998, pp. 555-559.

[41] M. Mignotte, "An adaptive segmentation-based regularization term for image restoration," in IEEE Int. Conf. Image Processing, Sep. 1998.

[42] K. May, T. Stathaki, A. G. Constantinides, and A. K. Katsaggelos, "Iterative determination of local bound constraints in iterative image restoration," in Proc. IEEE Int. Conf. Image Processing, vol. 2, Oct. 1998, pp. 833-836.

[43] R. Molina, A. K. Katsaggelos, J. Mateos, A. Hermoso, and C. A. Segall, "Restoration of severely blurred high range images using stochastic and deterministic relaxation algorithms in compound Gauss-Markov random fields," Pattern Recognit., vol. 33, no. 4, pp. 555-571, 2000.

[44] P. Charbonnier, L. Blanc-Feraud, G. Aubert, and M. Barlaud, "Deterministic edge-preserving regularization in computed imaging," IEEE Trans. Image Process., vol. 5, no. 12, pp. 298-311, Dec. 1997.

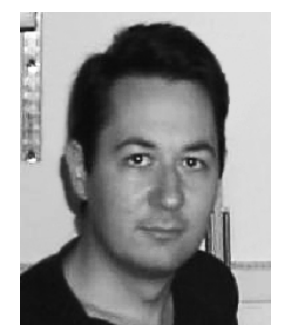

Max Mignotte received the DEA degree in digital signal, image, and speech processing from the INPG University, Grenoble, France, in 1993, and the Ph.D. degree in electronics and computer engineering from the University of Bretagne Occidentale (UBO) and the digital signal laboratory (GTS) of the French Naval Academy in 1998.

He was an INRIA Postdoctoral Fellow with the University of Montreal (DIRO), Montreal, QC, Canada, from 1998 to 1999 . He is currently with DIRO at the Computer Vision and Geometric Modeling Lab as an Assistant Professor (Professeur Adjoint) at the University of Montreal. He is also a member of LIO (Laboratoire de Recherche en Imagerie et Orthopedie, Centre de Recherche du CHUM, Hopital Notre-Dame) and Researcher at CHUM. His current research interests include statistical methods and Bayesian inference for image segmentation (with hierarchical Markovian, statistical templates, or active contour models), hierarchical models for high-dimensional inverse problems from early vision, parameters estimation, tracking, classification, shape recognition, deconvolution, 3-D reconstruction, and restoration problems. 